\title{
Airway observations during upper endoscopy predicting obstructive sleep apnea
}

\author{
Glenn Harvina, Eslam Alia , Amit Raina ${ }^{a}$, William Lelanda, Sabeen Abid ${ }^{b}, Z^{a}$ ahid Vahorab, \\ Hossein Movahed ${ }^{b}$, Sumyra Kachrub ${ }^{b}$, Rick Tee ${ }^{b}$
}

Brody School of Medicine at East Carolina University; Vidant Medical Center, USA

\section{Abstract} Background This pilot study examined airway characteristics during upper endoscopy to
determine who is at high risk for obstructive sleep apnea.

Methods Patients undergoing routine upper endoscopy were divided into 2 groups according to the Berlin Questionnaire (high and low risk for sleep disordered breathing). Patients underwent routine upper endoscopy using propofol sedation. The airway was then evaluated for no, partial, or complete collapse at the levels of the palate/uvula/tonsils, the tongue base, the hypopharynx, and the larynx. They were given a score of 0 for no collapse, 1 for partial collapse, and 2 for complete collapse. The score for each of these levels was added to give a total score or severity index. The larynx was also evaluated for lateral pharyngeal collapse (minimal, up to $50 \%,>50 \%$, or $100 \%$ ).

Results We found that patients with a partial obstruction at the level of the palate/uvula/tonsils, tongue base, hypopharynx, or larynx, or complete obstruction at any level more often had a positive Berlin questionnaire. Patients with a positive Berlin questionnaire were more often of increased weight (mean 197 vs $175 \mathrm{lbs}, \mathrm{P}=0.19)$, increased body mass index ( $31.2 \mathrm{vs} 27.42 \mathrm{~kg} / \mathrm{m}^{2}$, $\mathrm{P}=0.11$ ), increased neck circumference ( 36.7 vs $34.7 \mathrm{~cm}, \mathrm{P}=0.23$ ), and had a higher total airway score (2.61 vs $1.67, \mathrm{P}=0.09$ ).

Conclusions The results of our pilot study represent preliminary data regarding the use of upper endoscopy as a potential tool to evaluate patients for obstructive sleep apnea.

Keywords Upper endoscopy, sleep apnea, Berlin questionnaire, airway

Ann Gastroenterol 2016; 29 (3): 1-6

\section{Introduction}

Studies have noted that the pharynx is often passed by quickly or overlooked completely during routine gastrointestinal (GI) upper endoscopy [1], representing a missed opportunity for analysis of the airway. The ear, nose and throat (ENT) literature has well-documented the airway characteristics predictive of obstructive sleep apnea (OSA), and the current pilot study sought to apply the observation of these characteristics to

Departments of Gastroenterology a Brody School of Medicine at East Carolina University (Glenn Harvin, Eslam Ali, Amit Raina, William Leland); 'Vidant Medical Center (Sabeen Abid, Zaid Vahora, Hossein Movahed, Sumyra Kachru, Rick Tee), USA

Conflict of Interest: None

Correspondence to: Dr Glenn Harvin, Ass. Professor, Brody School of Medicine at East Carolina University, Greenville, NC 27834, USA, Tel.: +1 252744 5681, e-mail: harving@ecu.edu

Received 11 March 2016; accepted 9 May 2016; published online 10 June 2016

DOI: http://dx.doi.org/10.20524/aog.2016.0054 patients undergoing routine GI upper endoscopy. The aim of the study was to define airway characteristics that might be observed during routine upper endoscopy and can predict who is at high risk for OSA. These patients may then be referred for a sleep study.

OSA affects $2-4 \%$ of the population [2]. Around $92 \%$ of females and $82 \%$ males with OSA are undiagnosed [3]. OSA has been associated with an increased cardiovascular mortality_ and risk for stroke $[4,5]$. Various screening tools to identify patients at risk for OSA have been used, including the Berlin questionnaire, the American Society of Anesthesiologists (ASA) checklist, the STOP questionnaire and STOP-BANG [6,7]. The Berlin Questionnaire has been considered to be one of the best methods to identify patients likely to have OSA. One study placed its sensitivity at $86 \%$ and its specificity at $77 \%$ with a positive predictive value of $89 \%$ for predicting respiratory disturbance index of greater than 5 in patients in the highrisk group on the Berlin questionnaire [8]. Similar to these screening tests, various classification systems have been used in the ENT literature to define levels of obstruction observed as part of sleep endoscopy. These include the VOTE classification, Croft and Pringle, and the Novel Grading system [9-11]. 
Croft and Pringle described this technique to directly observe endoscopically the site or sites of obstruction of the upper airway in the sleeping patient following sedation [10]. Druginduced sleep endoscopy (DISE) has been studied in the ENT literature in defining upper airway obstruction [10,12]._DISE entails endoscopic examination of the upper airway after a patient is sedated (using pharmacologic agents) to reproduce upper airway behavior similar to natural sleep [11]. DISE has been shown to have a high interrater reliability for the presence of obstruction at the palate (K value 0.76 ) and hypopharynx (K value 0.79) [13]. Bachar et al described a "Novel Grading System for Quantifying Upper Airway obstruction on Sleep Endoscopy" [9]. The nose/nasopharynx, palate/uvula/tonsils, tongue base, hypopharynx and larynx were evaluated for obstruction and given a severity grade for each level, and the numeric values ( 0 , no obstruction; 1 , partial obstruction; 2 , complete obstruction) from each level were added to yield a severity index (SI) [9]. Another study showed that of patients who snored greater than $10 \mathrm{sec}$ during their screening colonoscopies, all were noted to have OSA [14].

\section{Patients and methods}

The study was approved by the East Carolina University and Medical Center Institutional Review Board. Adult patients having an upper endoscopy for routine indications at the East Carolina University outpatient endoscopy center between the dates November 3, 2014 and February 12, 2015 were eligible to participate in the study if they did not carry a diagnosis of OSA or had not had a prior sleep study. Other exclusion criteria comprised our standard exclusion criteria for the outpatient endoscopy center including body mass index (BMI) $>52 \mathrm{~kg} / \mathrm{m}^{2}$, age $<18$ years, cardiac defibrillator, home oxygen use, and ASA class 4 patients. Patients with prior surgery for head and neck cancer or a tracheostomy were also not eligible as this was felt to have distorted the normal anatomy. Patients having a colonoscopy at the same time as their upper endoscopy were eligible for the study. In these patients, the upper endoscopy was performed prior to the colonoscopy.

Informed consent was obtained from all the patients. All patients had a determination of their height, weight, BMI, Mallampati score, and neck circumference and whether they had a history of hypertension. The Mallampati was calculated by both the gastroenterologist and the nurse anesthetist, and a consensus was reached prior to performing the procedure. All patients were administered the Berlin questionnaire prior to the procedure by either the attending $(\mathrm{GH}, \mathrm{EA}, \mathrm{WL})$ or the research fellow. Patients were determined to be high risk if they met at least 2 of the 3 symptom categories. For calculation of the Berlin questionnaire, if the patient was negative by category 2 or 3 , and if they did not know whether they snored, they were given an equivocal result. All the patients were then divided into 2 groups according to the Berlin Questionnaire (high likelihood for sleep disordered breathing and low risk for sleep disordered breathing). The group determined to have a high likelihood of sleep disordered breathing (positive result on the
Berlin questionnaire) were recommended a follow up with the primary care physician to consider a sleep study per standard medical care.

All patients had the upper endoscopy performed under standard monitoring to include blood pressure, continuous heart rate and oxygen saturation monitoring. Nurse anesthetist- administered propofol was used for all cases. All patients had $2 \mathrm{~L}$ of oxygen per nasal cannula with the patients lying in the left lateral decubitus position. A neutral neck position was maintained during the procedure and evaluation of the airway. Any drops in oxygen saturation $<92 \%$ were noted and were treated with a head-tilt method. Patients were also observed for the presence of snoring for $>10 \mathrm{sec}$.

The upper airway was evaluated by the attending gastroenterologist performing the upper endoscopy during the insertion of the scope and was evaluated with a neutral neck position. The airway was evaluated at the level of the palate/uvula/tonsils, the tongue base, the hypopharynx, and the larynx. These levels were evaluated for no collapse, partial collapse, or complete collapse, and were given a score of 0 for no collapse, 1 for partial collapse, and 2 for complete collapse (Figs. 1 and 2) for pictures of these findings) according to Bachar et al [9]. Partial obstruction was defined as "narrowing or intermittent collapse" of the airway, and complete obstruction was defined as "complete blockage" of the airway passage [9]. The score for each of these levels was added to give a total score or SI as described previously by Bachar et al. The larynx was also evaluated for lateral pharyngeal collapse (minimal, up to $50 \%,>50 \%$, or $100 \%$ ).

\section{Statistical analysis}

Data were entered manually, and statistical analysis was performed using SAS Version 9.1 (SAS Institute, Cary, NC). Descriptive statistics were performed using standard methods. Chi-square test and Fischer's exact test were used to test direct association between individual obstruction measures and drop in oxygen saturation $<92 \%$ with the results of the Berlin questionnaire. A two-sample $t$-test was used to test direct association between height, weight, BMI, neck circumference, total obstruction score, and the Berlin questionnaire. Logistic regression was used to test the combined effect of multiple predictors to the Berlin questionnaire.

\section{Results}

A total of 35 patients were enrolled and completed the study, mean age was 51 (range 23-83) years. Thirty patients underwent an upper endoscopy only and 5 patients underwent an upper endoscopy followed by a colonoscopy. The baseline demographics are listed below in Table 1. The ASA classification results were as follows: 6 patients ASA class 1 (17.14\%), 17 patients ASA class 2 (48.57\%), and 12 ASA class 3 (34.29\%). There were no ASA class 4 patients as they were not eligible for our outpatient endoscopy facility, and were excluded from the study. 


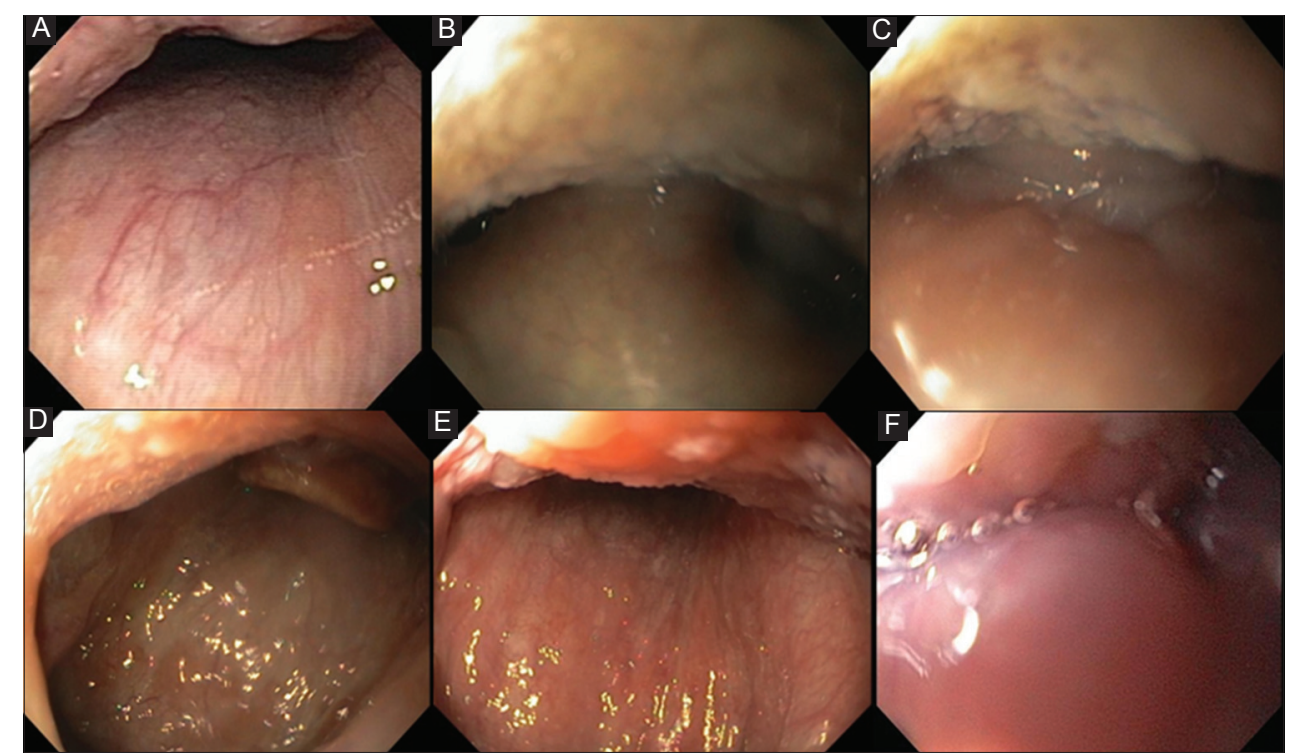

Figure 1 Palate/uvula/tonsils: (A) no obstruction, (B) partial obstruction, (C) complete obstruction. Tongue base: (D) no obstruction, (E) partial obstruction, (F) complete obstruction

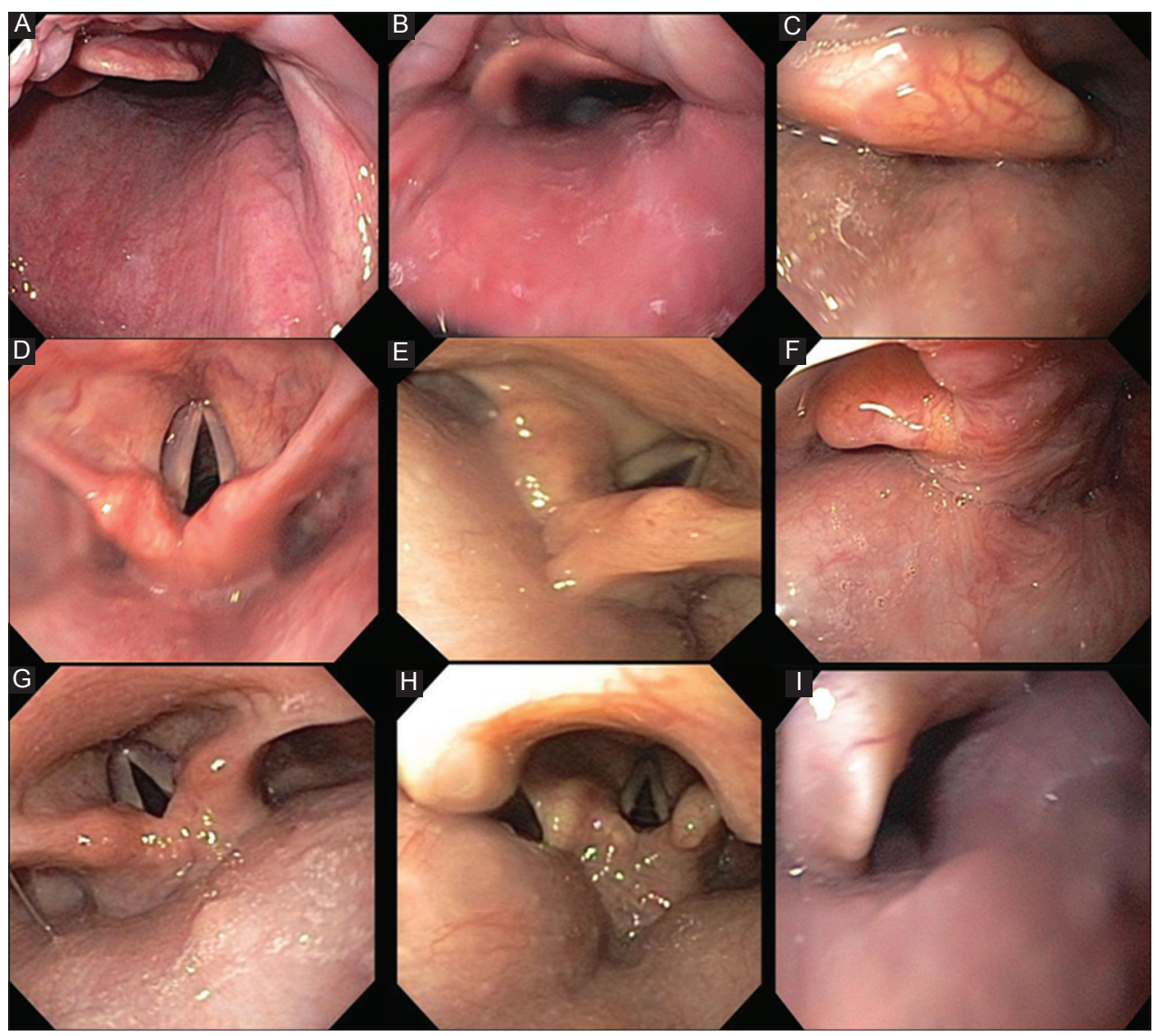

Figure 2 Hypopharynx: (A) no obstruction, (B) partial obstruction, (C) complete obstruction. Larynx: (D) no obstruction, (E) partial obstruction, (F) complete obstruction. Lateral pharyngeal collapse: (G) minimal, (H) up to $50 \%$, (I) $>50 \%$

The indications for the procedures included dysphagia $(\mathrm{n}=10)$, abdominal pain $(\mathrm{n}=7)$, gastroesophageal reflux disease ( $n=6$ patients), esophageal variceal screening $(n=3)$, follow up of gastric ulcers $(n=2)$, nausea and vomiting $(n=2)$, abnormal abdominal imaging $(n=1)$, follow up of small bowel lymphoma $(n=1)$, iron deficiency anemia $(n=1)$, and atypical 
chest pain $(n=1)$, and diarrhea $(n=1)$. Twenty-one patients had hypertension $(60 \%)$ and 14 patients did not carry a diagnosis of hypertension (40\%). The baseline Mallampati results can be seen in Table 2. Airway obstruction at different levels can be visualised in Table 3.

Eighteen patients had a positive Berlin questionnaire (51.4\%), 15 patients had a negative Berlin questionnaire (42.9\%), and 2 patients had an equivocal result (5.71\%). Six of the 35 patients had snoring greater than $10 \mathrm{sec}(17.1 \%)$, and $13 / 35$ patients (37.1\%) had a drop in oxygen saturation less than $92 \%$.

The Berlin questionnaire was positive in $64 \%$ patients with a partial obstruction at the level of the palate/uvula/ tonsils versus $47.4 \%$ of patients with no obstruction at this level $(\mathrm{P}=0.33)$. The Berlin questionnaire was positive in $61 \%$ patients with partial obstruction at the level of the tongue base versus $40 \%$ patients with no obstruction at this level $(\mathrm{P}=0.27)$. The Berlin questionnaire was positive in $61 \%$ patients with partial obstruction at the level of the hypopharynx versus $47 \%$ of patients with no obstruction at this level $(\mathrm{P}=0.41)$. The Berlin questionnaire was positive in $73 \%$ patients with a partial obstruction at the level of the larynx versus $45 \%$ patients with no obstruction at the level of the larynx $(\mathrm{P}=0.14)$. The Berlin questionnaire was positive in $80 \%$ patients with complete obstruction at any level versus $50 \%$ of patients with no evidence of complete obstruction at any level $(\mathrm{P}=0.21)$. The Berlin questionnaire was positive in $67 \%$ patients with a drop in oxygen saturation $<92 \%$ versus $48 \%$ of patients with a negative Berlin questionnaire $(\mathrm{P}=0.29)$.

Patients with a positive Berlin questionnaire were more often of increased weight (mean 197 vs $175 \mathrm{lbs}, \mathrm{P}=0.19$ ), increased BMI (31.2 vs $\left.27.42 \mathrm{~kg} / \mathrm{m}^{2}, \mathrm{P}=0.11\right)$, increased neck circumference ( 36.7 vs $34.7 \mathrm{~cm}, \mathrm{P}=0.23$ ), and had a higher total airway score (2.61 vs $1.67, \mathrm{P}=0.09$ ). Patients with a positive Berlin questionnaire more often had a drop in oxygen saturation $<92 \%$ ( 66.7 vs $47.6 \%, \mathrm{P}=0.29$ ). Four of six patients with snoring $>10 \mathrm{sec}$ during the upper endoscopy had a positive Berlin questionnaire $(\mathrm{P}=0.66)$.

Table 1 Summary of patient measurements

\begin{tabular}{lcccc}
\hline & Mean & Std Dev & Minimum & Maximum \\
\hline Age (years) & 51.4 & 16.4 & 23.0 & 83.0 \\
Height (inches) & 66.5 & 4.7 & 57.0 & 78.0 \\
Weight $(\mathrm{lbs})$ & 190.5 & 51.0 & 116.0 & 298.0 \\
BMI $\left(\mathrm{kg} / \mathrm{m}^{2}\right)$ & 30.1 & 7.4 & 18.0 & 51.2 \\
$\begin{array}{l}\text { Neck } \\
\text { circumference }(\mathrm{cm})\end{array}$ & 35.9 & 4.9 & 27.0 & 49.0 \\
\hline
\end{tabular}

Table 2 Overall distribution of Mallampati class

\begin{tabular}{lcc}
\hline Mallampati & Frequency & Percent \\
\hline 1 & 12 & 34.3 \\
2 & 9 & 25.7 \\
3 & 13 & 37.1 \\
4 & 1 & 2.9 \\
\hline
\end{tabular}

\section{Discussion}

Around $92 \%$ of females and $82 \%$ males with OSA are undiagnosed [3]. This underscores the need to develop novel methods to identify patients at risk for sleep apnea. Taking a cue from the findings of prior sleep endoscopy studies we have attempted to assess the feasibility of using a routine upper endoscopy encounter as a setting to screen patients at risk for OSA. Assessing the upper airway during a routine upper endoscopy is safe, quick, and should not add much time to the overall procedure, although it does have the added risk of sedation over a typical sleep study. Some earlier studies have demonstrated that the pharynx is often passed by quickly or overlooked completely during routine upper GI endoscopy [1]. While our findings did not reach statistical significance, we did see a trend $(\mathrm{P}=0.21)$ towards

Table 3 Airway obstruction at different levels

\begin{tabular}{|c|c|c|}
\hline & Frequency & Percent \\
\hline \multicolumn{3}{|l|}{ Palate/uvula/tonsils } \\
\hline 0 (no obstruction) & 19 & 54.3 \\
\hline 1 (partial obstruction) & 13 & 37.1 \\
\hline 2 (complete obstruction) & 3 & 8.6 \\
\hline \multicolumn{3}{|l|}{ Tongue base } \\
\hline 0 (no obstruction) & 10 & 28.6 \\
\hline 1 (partial obstruction) & 19 & 54.3 \\
\hline 2 (complete obstruction) & 6 & 17.1 \\
\hline \multicolumn{3}{|l|}{ Hypopharynx } \\
\hline 0 (no obstruction) & 15 & 42.9 \\
\hline 1 (partial obstruction) & 19 & 54.3 \\
\hline 2 (complete obstruction) & 1 & 2.9 \\
\hline \multicolumn{3}{|l|}{ Larynx } \\
\hline 0 (no obstruction) & 22 & 62.9 \\
\hline 1 (partial obstruction) & 13 & 37.1 \\
\hline 2 (complete obstruction) & 0 & 0 \\
\hline \multicolumn{3}{|l|}{ Lateral pharyngeal collapse } \\
\hline $1=$ minimal & 21 & 60 \\
\hline $2=$ up to $50 \%$ & 5 & 14.3 \\
\hline $3>50 \%$ collapse & 9 & 25.7 \\
\hline \multicolumn{3}{|l|}{ Total airway score } \\
\hline 0 & 7 & 20 \\
\hline 1 & 5 & 14.3 \\
\hline 2 & 7 & 20 \\
\hline 3 & 6 & 17.1 \\
\hline 4 & 6 & 17.1 \\
\hline 5 & 2 & 5.7 \\
\hline 6 & 1 & 2.9 \\
\hline 7 & 1 & 2.9 \\
\hline
\end{tabular}


a positive Berlin questionnaire in patients with a partial or complete collapse at various levels. Larger, well-powered studies will have to be performed to assess this further, and, if confirmed, this will make a case for screening all patients at the time of an endoscopic procedure. This could be during the upper GI endoscopy, as was the case in this study, or at the time of a screening colonoscopy. This approach would greatly increase the screening of the population for OSA and ensure that a large portion of the population is screened at the age of 50 .

In a study by Khiani et al, 39\% of patients undergoing routine upper endoscopy at a university-based outpatient center scored high risk for OSA as defined by the Berlin questionnaire [15]. A separate study by Mador et al at a Veterans Affairs outpatient endoscopy center also showed that $39 \%$ of patients scored high risk for OSA as defined by the Berlin questionnaire [16]. In our study, $51.43 \%$ of patients scored positive on the Berlin questionnaire, and thus were considered to be at high risk for OSA. This confirms the prior data showing that many patients with OSA are not being screened and identified, and this study illustrated that a large portion of patients presenting to our university-based outpatient endoscopy center likely have undiagnosed OSA.

Bachar et al calculated the SI for OSA based on different possible sites of obstruction in the upper airway, and noted that the SI correlated with the respiratory disturbance index and the BMI, and predicted disease severity with $65 \%$ accuracy [9]. We used a similar SI and grading system but eliminated the nose/nasopharynx, as this part is not seen on a typical upper endoscopy. It is important to discuss the fact that our study did not show a statistical association between the airway characteristics and the results of the Berlin questionnaire. Although our study showed an increased risk of airway collapse on upper endoscopy for patients determined to be at high risk for sleep apnea as defined by the Berlin questionnaire $(\mathrm{P}=0.21)$, this did not reach statistical significance. This is likely due to the low volume of patients (only 35) enrolled in the study, and perhaps with increased recruitment, this value might become significant. This study serves as a pilot study, and, ideally, we would have liked to have enrolled additional patients and to have referred each patient for a sleep study. We referred patients with a positive Berlin questionnaire to their primary care to consider a sleep study per standard care.

It has been noted in the ENT literature that an increased respiratory disturbance index correlated with the number of sites of obstruction on sleep endoscopy, although this was not seen in our study with the caveat we were using the Berlin questionnaire rather than a sleep study [17]. Ravesloot noted that multilevel collapse, complete collapse and a tongue-base collapse on DISE are associated with high apnea hypopnea values [18]. Steinhart noted an increased collapsibility in the area of the tongue base and stronger collapse of the upper airway in patients with OSA [19]. We noted that patients with a larger neck circumference more often had a positive Berlin questionnaire $(\mathrm{P}=0.22)$, as did patients with an increased BMI
$(\mathrm{P}=0.13)$. Some have found that neck circumference is a better predictor of obstruction than BMI [20,21]. Others have found that BMI but not neck circumference is a better predictor [22]. Patients with a positive Berlin questionnaire more often had a drop in oxygen saturation $(\mathrm{P}=0.24)$. Other studies have noted more oxygen desaturations in patients with high risk for OSA [23], although Khiani and Mador did not find an increased risk of desaturations $[15,16]$. Although propofol has been shown to affect sleep architecture, the apnea-hypopnea index and oxygen saturation is not affected with DISE with propofol sedation [24].

Overall, analysis of the upper airway at the time of a routine upper GI endoscopy suggests patients who are at high risk for sleep apnea. This opens the way for further research that would compare endoscopic analysis of the upper airway to the gold standard for the diagnosis of sleep apnea, a sleep study.

\section{Summary Box}

\section{What is already known:}

- The majority of patients with obstructive sleep apnea are undiagnosed

- Studies have noted that the pharynx is often passed by quickly or overlooked completely during routine upper gastrointestinal endoscopy representing a missed opportunity for analysis of the airway

- Various classification systems have been used in the ear, nose and throat literature to define levels of obstruction observed as part of sleep endoscopy

\section{What the new findings are:}

- Assessing airway characteristics at the time of a routine upper endoscopy is feasible

- Analysis of the upper airway at the time of a routine upper gastrointestinal endoscopy suggests patients who are at high risk for sleep apnea

- We did see a trend towards a positive Berlin questionnaire in patients with a partial or complete collapse at various levels

- A large portion of patients presenting to our university-based outpatient endoscopy center likely have undiagnosed sleep apnea and are not being screened and identified by routine methods

\section{Acknowledgments}

Special thanks to Dr. Sherif El-Behiry who assisted with the design of the study and Dr. Qiang Wu who performed the statistical analysis. 


\section{References}

1. Emura F, Baron TH, Gralnek IM. The pharynx: examination of an area too often ignored during upper endoscopy. Gastrointest Endosc 2013;78:143-149.

2. Young T, Palta M, Dempsey J, et al. The occurrence of sleepdisordered breathing among middle-aged adults. $N$ Engl J Med 1993;328:1230-1235.

3. Chung F, Subramanyam R, Liao P, et al. High STOP-Bang score indicates a high probability of obstructive sleep apnoea. $\mathrm{Br} J$ Anaesth 2012;108:768-775.

4. Koskenvuo M, Kaprio J, Telakivi T, et al. Snoring as a risk factor for ischaemic heart disease and stroke in men. Br Med J (Clin Res Ed) 1987;294:16-19.

5. Poceta JS, Loube DI, Kellgren EL, et al. Mortality in obstructive sleep apnea: Association with Impaired Wakefulness. Sleep Breath 1999;3:3-8.

6. Chung F, Yegneswaran B, Liao P, et al. Validation of the Berlin questionnaire and American Society of Anesthesiologists checklist as screening tools for obstructive sleep apnea in surgical patients. Anesthesiology 2008;108:822-830.

7. Chung F, Yegneswaran B, Liao P, et al. STOP questionnaire: a tool to screen patients for obstructive sleep apnea. Anesthesiology 2008; 108:812-821.

8. Netzer NC, Stoohs RA, Netzer CM, et al. Using the Berlin Questionnaire to identify patients at risk for the sleep apnea syndrome. Ann Intern Med 1999;131:485-491.

9. Bachar G, Nageris B, Feinmesser R, et al. Novel grading system for quantifying upper-airway obstruction on sleep endoscopy. Lung 2012;190:313-318.

10. Croft CB, Pringle M. Sleep nasendoscopy: a technique of assessment in snoring and obstructive sleep apnoea. Clin Otolaryngol Allied Sci 1991;16:504-509.

11. Kezirian EJ, Hohenhorst W, de Vries N. Drug-induced sleep endoscopy: the VOTE classification. Eur Arch Otorhinolaryngol 2011;268:1233-1236.

12. Soares D, Folbe AJ, Yoo G, et al. Drug-induced sleep endoscopy vs awake Muller's maneuver in the diagnosis of severe upper airway obstruction. Otolaryngol Head Neck Surg 2013;148:151-156.

13. Kezirian EJ, White DP, Malhotra A, et al. Interrater reliability of drug-induced sleep endoscopy. Arch Otolaryngol Head Neck Surg 2010;136:393-397.

14. Sharara AI, El Zahabi L, Maasri K, et al. Persistent snoring under conscious sedation during colonoscopy is a predictor of obstructive sleep apnea. Gastrointest Endosc 2010;71:1224-1230.

15. Khiani VS, Salah W, Maimone S, et al. Sedation during endoscopy for patients at risk of obstructive sleep apnea. Gastrointest Endosc 2009;70:1116-1120.

16. Mador MJ, Abo Khamis M, Nag N, et al. Does sleep apnea increase the risk of cardiorespiratory complications during endoscopy procedures? Sleep Breath 2011;15:393-401.

17. Bachar G, Feinmesser R, Shpitzer T, et al. Laryngeal and hypopharyngeal obstruction in sleep disordered breathing patients, evaluated by sleep endoscopy. Eur Arch Otorhinolaryngol 2008;265:1397-1402

18. Ravesloot MJ, de Vries N. One hundred consecutive patients undergoing drug-induced sleep endoscopy: results and evaluation. Laryngoscope 2011;121:2710-2716.

19. Steinhart H, Kuhn-Lohmann J, Gewalt K, et al. Upper airway collapsibility in habitual snorers and sleep apneics: evaluation with drug-induced sleep endoscopy. Acta Otolaryngol 2000;120:990-994.

20. Davies RJ, Ali NJ, Stradling JR. Neck circumference and other clinical features in the diagnosis of the obstructive sleep apnoea syndrome. Thorax 1992;47:101-105.

21. Hoffstein V, Mateika S. Differences in abdominal and neck circumferences in patients with and without obstructive sleep apnoea. Eur Respir J 1992;5:377-381.

22. Deegan PC, McNicholas WT. Predictive value of clinical features for the obstructive sleep apnoea syndrome. Eur Respir J 1996;9:117-124.

23. Cote GA, Hovis CE, Hovis RM, et al. A screening instrument for sleep apnea predicts airway maneuvers in patients undergoing advanced endoscopic procedures. Clin Gastroenterol Hepatol 2010;8:660-665 e1.

24. Rabelo FA, Kupper DS, Sander HH, et al. Polysomnographic evaluation of propofol-induced sleep in patients with respiratory sleep disorders and controls. Laryngoscope 2013;123:2300-2305. 